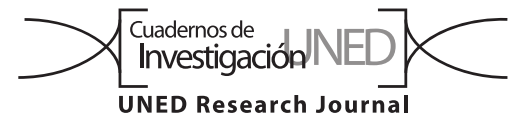

\title{
El armadillo, Cabassous centralis (Cingulata: Chlamyphoridae) en agroecosistemas con café de Costa Rica
}

\section{Ronald J. Sánchez-Brenes ${ }^{1}$ \& Javier Monge ${ }^{2}$}

1. Universidad Nacional Costa Rica, Sede Regional Chorotega, Centro Mesoamericano de Desarrollo Sostenible del Trópico Seco (CEMEDE-UNA), Nicoya, Costa Rica; ronald.sanchez.brenes@una.cr, (iD https://orcid.org/0000-0002-6979-1336

2. Universidad de Costa Rica, Facultad de Ciencias Agroalimentarias, Escuela de Agronomía, Centro de Investigación en Protección de Cultivos, Instituto de Investigaciones Agronómicas, San José, Costa Rica; javier.monge@ucr.ac.cr, (iD https://orcid.org/0000-0003-1530-5774

Recibido 09-VIII-2019 • Corregido 11-IX-2019 • Aceptado 30-IX-2019

DOI: https://doi.org/10.22458/urj.v11i3.2724

\begin{abstract}
The armadillo, Cabassous centralis (Cingulata: Chlamyphoridae) in a Costa Rican coffee agro-ecosystem". Introduction: The rare Cabassous centralis armadillo is classified as a Data Deficient species found from Mexico to northern South America). Objective: To expand the ecological distribution of $C$. centralis. Methods: We placed four trap cameras in strategic sites such as food sites, burrows, bodies of water and transition to the secondary forest, in San Ramón, Costa Rica. Results: We obtained a record of $C$. centralis in the transition between coffee plantation and secondary forest. Conclusion: This new finding near a coffee plantation adds to the list of habitats it occupies and can help in its conservation.
\end{abstract}

Key words: Agroecosystems, naked-tailed armadillo, Cingulata, coffee crops, armadillo.
RESUMEN: Introducción: El armadillo Cabassous centralis se clasifica como una especie con Datos Insuficientes que se encuentra desde México hasta el norte de América del Sur. Objetivo: Ampliar la distribución ecológica de $C$. centralis. Métodos: Colocamos cuatro cámaras trampa en sitios estratégicos como fuentes de alimentación, madrigueras, cuerpos de agua y transición al bosque secundario, en San Ramón, Costa Rica. Resultados: Obtuvimos un registro de $C$. centralis en la transición entre la plantación de café y el bosque secundario. Conclusión: Este nuevo hallazgo cerca de un cafetal se suma a la lista de hábitats que ocupa y puede ayudar en su conservación.

Palabras clave: Agroecosistemas, armadillo cola de trapo, Cingulata, cultivo de café, armadillo Zopilote.
El armadillo zopilote Cabassous centralis (Miller, 1899) pertenece al orden Cingulata y a la familia Chlamyphoridae (Delsuc et al., 2016). Su distribución va desde el sureste de México hasta el noreste de Suramérica (Colombia, Venezuela y Ecuador) (Carrillo, Wong, \& Sáenz, 2002; Hayssen, Ortega, Morales-Leiva, \& Martínez-Mendez, 2012; Chacón-Pacheco, BallesterosCorrea, \& Racero-Casarrubia, 2019).

Este armadillo se puede encontrar en hábitats como bosques mesófilos, secos y sabanas (Hayssen et al., 2012; UICN, 2019). Actualmente se observa en lugares degradados, ya que es amenazado por el desarrollo comercial, residencial, agricultura, ganadería, acuicultura, caza y carreteras (Aguiar, 2004; Cruz-Rodríguez et al., 2011; Contreras \& Yanguez, 2017; UICN, 2019). Las características generales de $C$. centralis que lo distingue de los otros armadillos es su tamaño pequeño, cuerpo más aplanado, con una cola esbelta y corta, la cual tiene placas delgadas ampliamente espaciadas, de color gris rosado (Reid, 2009; Hayssen et al., 2012).

Su comportamiento es nocturno, terrestre y solitario (Emmons \& Feer, 1990; Gardner, Wetzel, Redford, \& Eisenberg, 2007). La mayor parte del tiempo pasa bajo tierra en túneles (Cuarón, 2005). C. centralis camina sobre las puntas de sus garras delanteras, cuando excava gira su cuerpo de modo que la parte delantera actúa como si fuera un barreno (Wetzel, 1980). C. centralis posee cinco dedos y la tercera garra es notablemente grande (Cuarón, 2005; Mendoza-Durán, 2005), además, puede nadar (Ingles, 1953) y tienen solo una cría por parto (RodríguezMahecha, Rueda-Almonacid, \& Hinojosa, 2008). 
La información de C. centralis es escasa, su tendencia poblacional actual desconocida y se cataloga como especie "rara" (Carrillo et al., 2002; Hayssen et al., 2012; UICN, 2019). Esta falta de información limita el conocimiento sobre sus requerimientos de hábitat, periodo de actividad, la interacción de este armadillo con otras especies y las estrategias para conservarlo (Figueroa-DeLeón, Naranjo, \& Santos-Moreno, 2016).

En Costa Rica están presentes dos especies de armadiIlo, el de nueve bandas, Dasypus novemcinctus que pertenece a la familia Dasypodidae y el armadillo zopilote Cabassous centralis (Carrillo \& Wong, 1992). C. centralis se ha observado más en bosques de tierras bajas que en bosques nubosos arriba de los $1500 \mathrm{msnm}$ (Genoways \& Timm, 2003). Además, se incluyó en el Apéndice III de CITES, como una acción puntual de conservación (Cruz-Rodríguez et al., 2011). La investigación realizada en Rincón de Mora, San Ramón, Costa Rica, evidencia la aparición de $C$. centralis como el primer registro en un hábitat transformado como lo es un agroecosistema con cultivo de café y el sétimo registro de esta especie para Costa Rica.

\section{MATERIALES Y MÉTODOS}

El estudio se realizó en dos fincas colindantes con un área de 6,2ha, ubicadas en la comunidad de Rincón de Mora $\left(10^{\circ} 0^{\prime} 44^{\prime \prime} \mathrm{N}-84^{\circ} 7^{\prime} 28^{\prime \prime} \mathrm{O} \& 10^{\circ} 0^{\prime} 34^{\prime \prime} \mathrm{N}-84^{\circ} 7^{\prime} 28^{\prime \prime} \mathrm{O}\right)$ a 2,5km al sur del distrito de San Rafael, cantón de San Ramón, provincia de Alajuela. Las fincas objeto de estudio son agroecosistemas de café ubicados entre los 1200 y $1250 \mathrm{msnm}$, con pendientes de 15, 30 y 45\% (Sánchez \& Moya, 2018).

En esta zona se presenta una precipitación de $3461 \mathrm{~mm}$ por año, con una temperatura promedio de $20^{\circ} \mathrm{C}$ y una humedad relativa de $92 \%$ (CIGEFI, 2019). El uso de suelo de las fincas es 3,6ha en agroecosistema con café, 1,5ha forestal, $1266 \mathrm{~m}^{2}$ de cultivo de mora, un invernadero de $343 \mathrm{~m}^{2}$ y el área restante corresponde a construcciones. En el sitio de estudio se colocaron cuatro cámaras trampa Modelo Bushnell Natureview HD en sitios estratégicos como fuentes de alimentación, madrigueras, cuerpos de agua y transición con el bosque secundario (Fig. 1).

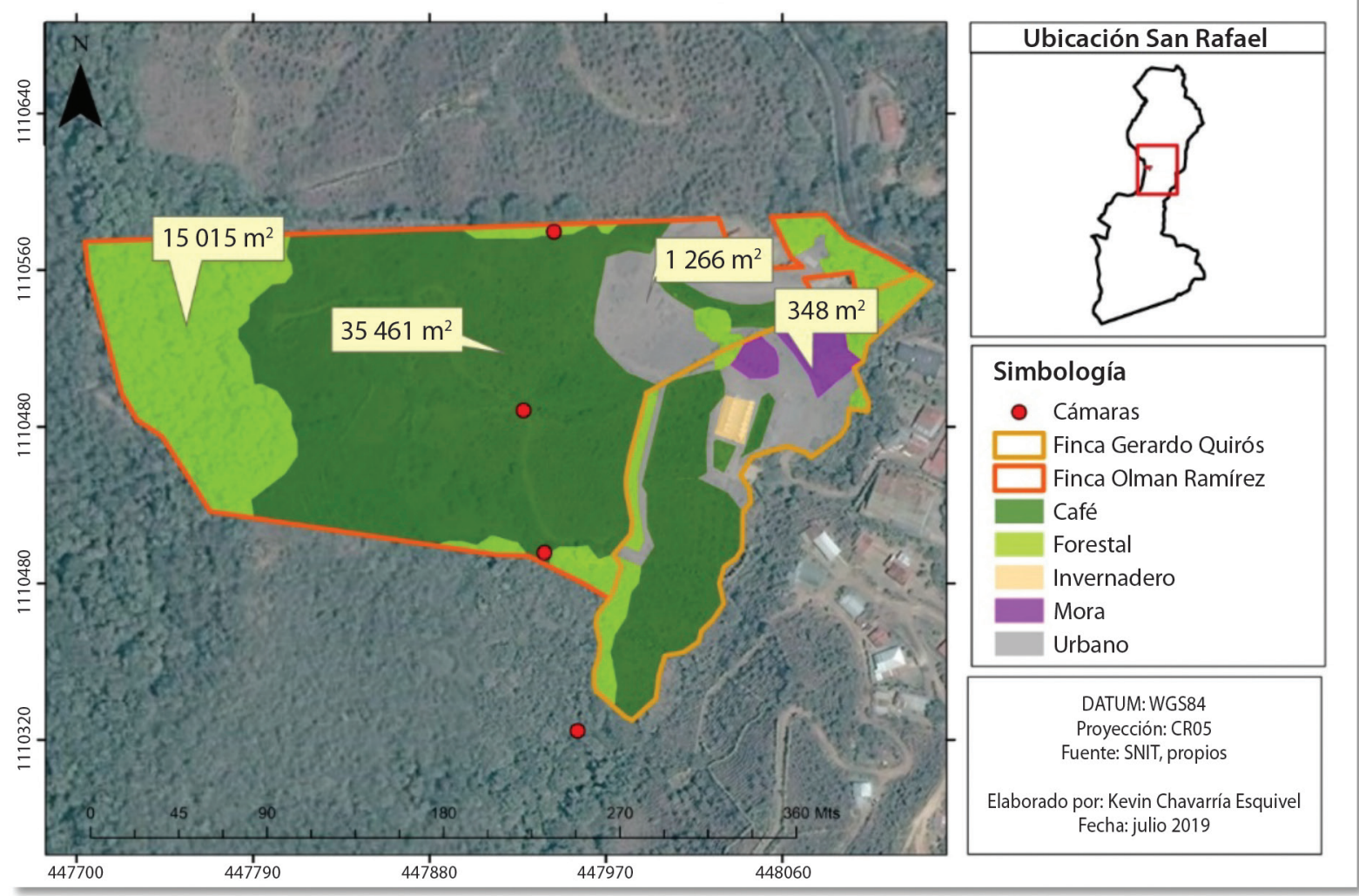

Fig. 1. Sitio de estudio y ubicación de cámaras trampa. 
Ética, conflicto de intereses y declaración de financiamiento: Los autores declaran haber cumplido con todos los requisitos éticos y legales pertinentes, tanto durante el estudio como en el manuscrito; que no hay conflictos de interés de ningún tipo, y que todas las fuentes financieras se detallan plena y claramente en la sección de agradecimientos. Asimismo, están de acuerdo con la versión editada final del documento. El respectivo documento legal firmado se encuentra en los archivos de la revista.

\section{RESULTADOS}

El presente estudio hace mención de un nuevo registro de $C$. centralis en la comunidad de Rincón de Mora, San Rafael, San Ramón, Alajuela en la segunda cámara trampa, ubicada de sur a norte, colocada en la transición entre el cultivo de café con sombra que presenta vegetación herbácea a una altura aproximada de $50 \mathrm{~cm}$ y el bosque secundario (Fig. 1) dos veces, el 21 de mayo de 2019 a la 01:42 h y el 25 de julio de 2019 a las 23:02 h. Con estos nuevos hallazgos se llega a un total de siete registros para Costa Rica (Cuadro 1).

En los videos se observa a este armadillo cavando, caminando sobre las puntas, se notan las placas delgadas espaciadas y un cuerpo más aplanado que lo diferencia de Dasypus novemcinctus, también presente en estos cafetales (Fig. 2, Fig. 3).
Con el fin de complementar la información sobre la distribución de $C$. centralis en Latinoamérica, se presen$\tan$ una serie de estudios en donde se ha reportado $C$. centralis siguiendo el orden de distribución (Cuadro 2, Cuadro 3 y Cuadro 4).

\section{DISCUSIÓN}

El armadillo $C$. centralis se puede encontrar en hábitats como bosques mesófilos, secos y sabanas (Hayssen et al., 2012; UICN, 2019), aunque también se observa en ambientes intervenidos, como en este caso en cultivos de café, lo cual puede deberse a la amenaza por el desarrollo comercial, residencial, agricultura, ganadería, acuicultura, caza y carreteras a sus hábitats naturales (Aguiar, 2004; Cruz-Rodríguez et al., 2011; UICN, 2019).

En cuanto a su comportamiento, Emmons y Feer (1990) y Gardner et al. (2007) indican que este edentado es nocturno, terrestre y solitario, lo cual dificulta a que se observado. Los registros en esta investigación confirman lo anterior dado que fueron a 01:42 $\mathrm{h} 21$ de mayo de 2019 y a las 23:02 h el 25 de julio de 2019.

En el sureste mexicano, existen 15 registros del armadillo zopilote Cabassous centralis (Miller 1899). La primera vez que se observó esta especie fue en 1984 y posteriormente, se confiscó una hembra adulta en 1986 (Cuarón et al., 1989). Entre 2007 y 2011 se registraron seis especímenes más (González-Zamora et al., 2011) Además

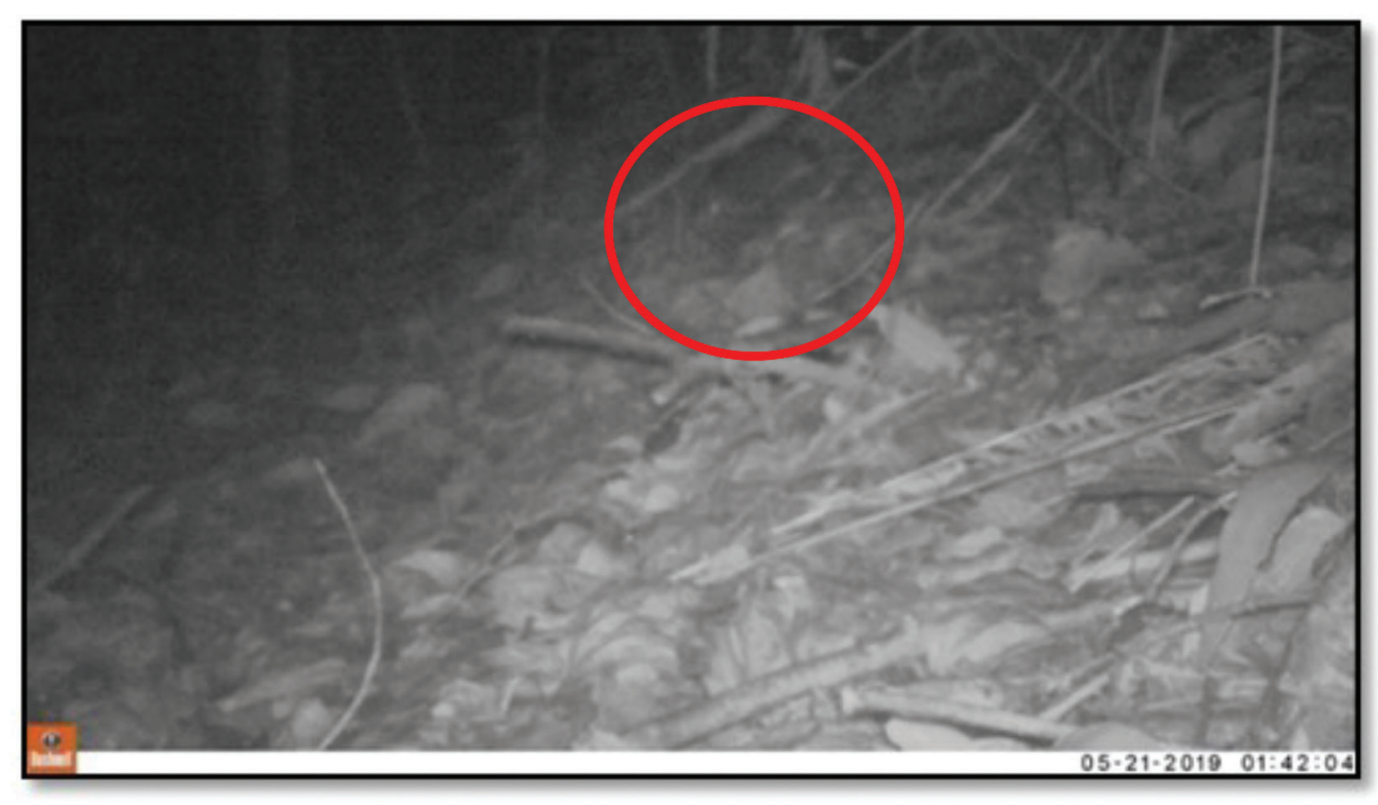

Fig. 2. Individuo de Cabassous centralis captado por cámara trampa el 21 de mayo de 2019, en Rincón de Mora, San Rafael, San Ramón, Alajuela, Costa Rica. 


\section{CUADRO 1}

Registros de Cabassous centralis en México

\begin{tabular}{|c|c|c|c|c|c|c|}
\hline País & Referencia & Fecha & Lugar & Hábitat & $\begin{array}{l}\text { Medio de } \\
\text { registro }\end{array}$ & $\begin{array}{l}\text { Condiciones } \\
\text { biofísicas }\end{array}$ \\
\hline \multirow{8}{*}{ 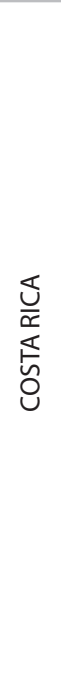 } & Hall, 1981 & 1981 & Costa Rica & Todo el país & - & - \\
\hline & Wilson, 1983 & 1983 & $\begin{array}{l}\text { Reserva Biológica } \\
\text { Monteverde y } \\
\text { Guanacaste }\end{array}$ & $\begin{array}{l}\text { Bosque nuboso y } \\
\text { bosque seco }\end{array}$ & - & - \\
\hline & $\begin{array}{c}\text { Timm, Wilson, } \\
\text { Clauson, LaVal, \& }\end{array}$ & Marzo 1986 & $\begin{array}{l}\text { Parque Nacional Braulio } \\
\text { Carrillo }\end{array}$ & Bosque nuboso & Observación & $200 \mathrm{msnm}$ \\
\hline & Vaughan, 1989 & $\begin{array}{l}\text { 1973, agosto } 1983 \\
\text { y diciembre } 1984\end{array}$ & $\begin{array}{c}\text { Estación Biológica } \\
\text { La Selva }\end{array}$ & Bosque húmedo & Observación & - \\
\hline & Carrillo \& Wong, 1992 & 14 de abril 1990 & $\begin{array}{l}\text { Parque Nacional Manuel } \\
\text { Antonio. Quepos }\end{array}$ & Bosque seco & $\begin{array}{l}\text { Espécimen } \\
\text { muerto }\end{array}$ & - \\
\hline & $\begin{array}{c}\text { Rodríguez \& } \\
\text { Chinchilla, } 1996\end{array}$ & - & $\begin{array}{l}\text { Estación Biológica La Selva, } \\
\text { Monteverde y Guanacaste }\end{array}$ & Bosque húmedo & - & - \\
\hline & Saénz \& Carrillo, 2009 & 20 de enero 2009 & $\begin{array}{c}\text { Reserva Forestal Pacuare y } \\
\text { Reserva Indígena Nairi Awari, } \\
\text { Siquirres }\end{array}$ & $\begin{array}{l}\text { Cobertura } \\
\text { Forestal Madura }\end{array}$ & $\begin{array}{l}\text { Cámara } \\
\text { Trampa }\end{array}$ & - \\
\hline & Este estudio & 21 de mayo 2019 & $\begin{array}{l}\text { Rincón de Mora, San Rafael, } \\
\text { San Ramón, Alajuela }\end{array}$ & $\begin{array}{l}\text { Agroecosistemas con } \\
\text { café }\end{array}$ & $\begin{array}{l}\text { Cámara } \\
\text { Trampa }\end{array}$ & $\begin{array}{c}1200-1250 \mathrm{msnm} \\
3461 \mathrm{~mm} / \mathrm{año} \\
20^{\circ} \mathrm{C}\end{array}$ \\
\hline
\end{tabular}

CUADRO 2

Registros de Cabassous centralis en México

\begin{tabular}{|c|c|c|c|c|c|c|}
\hline País & Referencia & Fecha & Lugar & Hábitat & $\begin{array}{l}\text { Medio de } \\
\text { registro }\end{array}$ & $\begin{array}{l}\text { Condiciones } \\
\text { Biofísicas }\end{array}$ \\
\hline \multirow{5}{*}{ 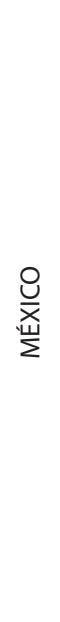 } & $\begin{array}{l}\text { Cuarón, March \& } \\
\text { Rockstroh, } 1989\end{array}$ & Agosto 1984 & Selva Lacandona, Chiapas & Bosque siempre verde & Reporte & - \\
\hline & $\begin{array}{c}\text { Cuarón et al., } \\
1989\end{array}$ & Octubre 1986 & $\begin{array}{l}\text { San Javier, Municipio de } \\
\text { Ocosingo, Chiapas }\end{array}$ & Bosque siempre verde & Individuo confiscado & - \\
\hline & $\begin{array}{c}\text { González-Zamora } \\
\text { et al., } 2011\end{array}$ & $\begin{array}{c}\text { Febrero } \\
\text { 2007- mayo } 2011\end{array}$ & $\begin{array}{c}\text { Zona de influencia Reserva } \\
\text { de la Biosfera Montes Azules, } \\
\text { Municipio de Marqués de } \\
\text { Comillas y Ocosingo, Selva } \\
\text { Lacandona, Chiapas }\end{array}$ & $\begin{array}{c}\text { Bosque tropical } \\
\text { perennifolio, pastizales } \\
\text { aledaños y vegetación } \\
\text { secundaria }\end{array}$ & $\begin{array}{l}\text { Atropello, huellas y } \\
\text { observación }\end{array}$ & - \\
\hline & $\begin{array}{l}\text { Figueroa-De-León } \\
\text { et al., } 2016\end{array}$ & $\begin{array}{l}\text { Setiembre 2013- } \\
\text { marzo } 2014\end{array}$ & $\begin{array}{c}\text { Zona de influencia de la } \\
\text { Reserva de la Biosfera Montes } \\
\text { Azules, Municipio de Marqués } \\
\text { de Comillas, Selva Lacandona, } \\
\text { Chiapas }\end{array}$ & $\begin{array}{c}\text { Bosque tropical } \\
\text { perennifolio, pastizales } \\
\text { aledaños y vegetación } \\
\text { secundaria }\end{array}$ & $\begin{array}{c}\text { Cámara trampa (Nueve } \\
\text { fotografías y cuatro } \\
\text { videos) }\end{array}$ & 146-148msnm \\
\hline & $\begin{array}{l}\text { Juárez-López et al., } \\
2017\end{array}$ & $\begin{array}{l}10 \text { de diciembre } \\
2016\end{array}$ & Portaceli, Palenque, Chiapas & $\begin{array}{l}\text { Bosque secundario en } \\
\text { crecimiento }\end{array}$ & Cámara trampa & $\begin{array}{c}100-500 \mathrm{msnm} \\
3000-3500 \mathrm{~mm} \\
26-28^{\circ} \mathrm{C}\end{array}$ \\
\hline
\end{tabular}

desde 2013-2014 se reportó C. centralis en 13 ocasiones (Figueroa-León et al., 2016).

Por su parte, en Centroamérica se tienen cinco registros de C. centralis. Nicaragua reportó por primera vez esta especie en 1854 (Bard, 1855), después en 1980 (Wetzel, 1980) y por último en 1998 (Woodman, 1998). Para 1982, Cuarón et al. (1989) hicieron el primer reporte en Guatemala de una hembra adulta, observado por
Peter Rockstroh. En 1986, ese colectó otra hembra adulta en este país. En el caso de Honduras se tiene un reporte en 2014 (Hoskins et al., 2018).

En Suramérica se cuenta con un total de 11 registros, todos en Colombia. No obstante, se menciona que su distribución puede estar en Venezuela y llegar hasta Ecuador (Carrillo, Wong, \& Sáenz, 2002; Hayssen et al., 2012; Chacón-Pacheco et al., 2019). El primer informe de 


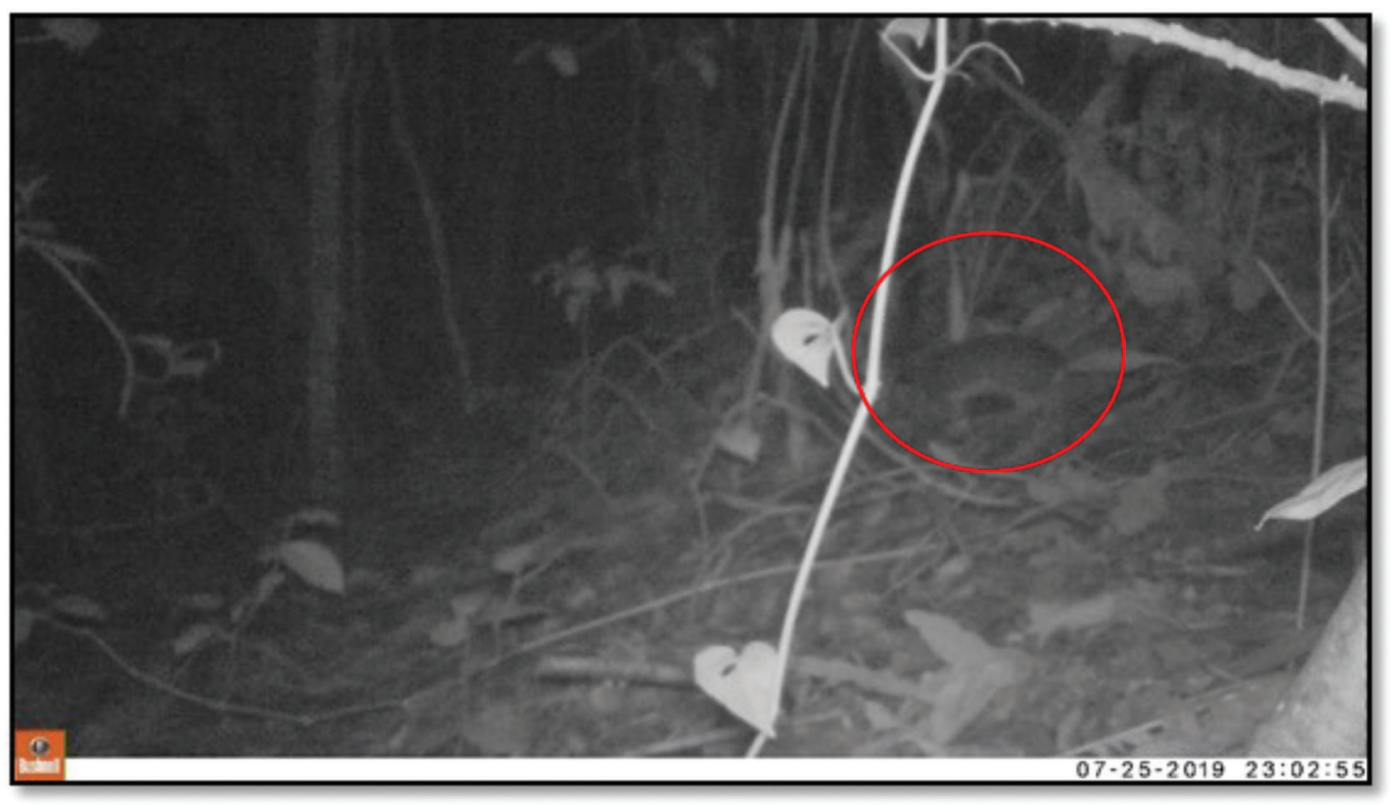

Fig. 3. Individuo de Cabassous centralis captado por cámara trampa el 25 de julio de 2019, en Rincón de Mora, San Rafael, San Ramón, Alajuela, Costa Rica.

CUADRO 3

Registros de Cabassous centralis en Centroamérica

\begin{tabular}{|c|c|c|c|c|c|c|}
\hline País & Referencia & Fecha & Lugar & Hábitat & Medio de registro & $\begin{array}{l}\text { Condiciones } \\
\text { biofísicas }\end{array}$ \\
\hline \multirow{3}{*}{ 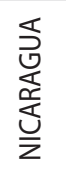 } & Bard, 1855 & 1854 & Costa Caribe & - & Observación & - \\
\hline & Wetzel, 1980 & 1980 & Managua & - & Reporte & - \\
\hline & Woodman, 1998 & 1998 & $\begin{array}{l}\text { San Ramón, Isla } \\
\text { Ometepe, Rivas }\end{array}$ & Bosque seco & Reporte & $\begin{array}{c}1600 \mathrm{~mm} / \mathrm{año} \\
27^{\circ} \mathrm{C}\end{array}$ \\
\hline \multirow{2}{*}{ 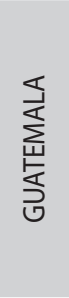 } & Cuarón et al., 1989 & $\begin{array}{l}\text { Octubre } \\
1986\end{array}$ & $\begin{array}{l}\text { Los Mariscos, } \\
\text { Izabal }\end{array}$ & $\begin{array}{l}\text { Plantaciones de caucho } \\
\text { (Helvea elastica) con } \\
\text { banano,cacao, maíz y parches } \\
\text { de bosque siempre verde. }\end{array}$ & Observación & - \\
\hline & Cuarón et al., 1989 & $\begin{array}{c}\text { Noviembre } \\
1986\end{array}$ & $\begin{array}{l}\text { Pueblo Nuevo, } \\
\text { Izabal }\end{array}$ & $\begin{array}{c}\text { Plantaciones de caucho } \\
\text { (Helvea elastica) con } \\
\text { banano,cacao, maíz y parches } \\
\text { de bosque siempre verde. }\end{array}$ & Colecta & - \\
\hline 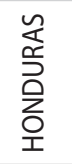 & Hoskins et al., 2018 & 2014 & $\begin{array}{l}\text { Parque Nacional el } \\
\text { Cusuco }\end{array}$ & Bosque nuboso & Cámara trampa & $\begin{array}{l}2240 \mathrm{msnm} \\
2788 \mathrm{~mm} / \mathrm{año} \\
12,9-20,2^{\circ} \mathrm{C}\end{array}$ \\
\hline
\end{tabular}


CUADRO 4

Registros de Cabassous centralis en Suramérica

\begin{tabular}{|c|c|c|c|c|c|c|}
\hline País & Referencia & Fecha & Lugar & Hábitat & $\begin{array}{l}\text { Medio de } \\
\text { registro }\end{array}$ & $\begin{array}{c}\text { Condiciones } \\
\text { biofísicas }\end{array}$ \\
\hline \multirow{8}{*}{ 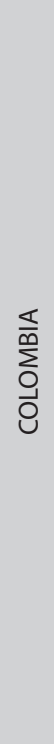 } & Bangs, 1900 & 1898 & Santa Marta, Magdalena & Costa Caribe & Captura & - \\
\hline & Allen, 1904 & 1900 & $\begin{array}{c}\text { Bonda y Valparaíso, Santa } \\
\text { Marta, Magdalena }\end{array}$ & Costa Caribe & Captura & - \\
\hline & $\begin{array}{l}\text { Corredor, et al., } \\
2004\end{array}$ & 2004 & $\begin{array}{c}\text { Santagueda, Palestina, } \\
\text { Manizales, Colombia }\end{array}$ & $\begin{array}{l}\text { Plantaciones viejas } \\
\text { de café con cultivos } \\
\text { de banano y yuca }\end{array}$ & Captura & $\begin{array}{c}1330 \mathrm{msnm} \\
2000 \mathrm{~mm} / \mathrm{año} \\
27^{\circ} \mathrm{C}\end{array}$ \\
\hline & $\begin{array}{c}\text { Díaz \& } \\
\text { Sánchez-Giraldo, } \\
2008\end{array}$ & $\begin{array}{l}13 \text { de } \\
\text { diciembre } \\
2005\end{array}$ & $\begin{array}{c}\text { Serranía de Las Baldías, } \\
\text { Corregimiento de San Félix, } \\
\text { Bello, Antioquia }\end{array}$ & - & $\begin{array}{c}\text { Restos (Cráneo, } \\
\text { mandíbula, } \\
\text { dientes y garras) }\end{array}$ & $3018 \mathrm{msnm}$ \\
\hline & $\begin{array}{l}\text { Rodríguez-Mahecha, } \\
\text { et al., } 2008\end{array}$ & - & $\begin{array}{c}\text { Santuario de Vida Silvestre } \\
\text { los Besotes, } \\
\text { Valledupar, Cesar }\end{array}$ & $\begin{array}{l}\text { Bosques secos y } \\
\text { húmedos }\end{array}$ & - & - \\
\hline & Cañon \& Trujillo, 2014 & $2009-2013$ & $\begin{array}{c}\text { El Cerrejón, Hatonuevo, } \\
\text { La Guajira }\end{array}$ & Complejo carbonífero & $\begin{array}{c}\text { Cámara Trampa y } \\
\text { Observación } \\
\text { Directa }\end{array}$ & - \\
\hline & $\begin{array}{l}\text { Cruz-Rodríguez, } \\
\text { et al., } 2011\end{array}$ & $\begin{array}{l}\text { Setiembre } 2010 \\
\text {-enero } 2011\end{array}$ & $\begin{array}{l}\text { Estación Primatológica } \\
\text { de Colosó, Tolú, Sucre }\end{array}$ & Bosque seco & $\begin{array}{c}\text { Cámara trampa y } \\
\text { huellas }\end{array}$ & $351 \mathrm{msnm}$ \\
\hline & $\begin{array}{l}\text { Chacón-Pacheco, } \\
\text { et al., } 2019\end{array}$ & $\begin{array}{c}\text { Diciembre } \\
\text { 2016-enero } 2017\end{array}$ & $\begin{array}{c}\text { Vereda el Venado, } \\
\text { Municipio de Tierralta, } \\
\text { Córdoba }\end{array}$ & $\begin{array}{l}\text { Bosque húmedo } \\
\text { tropical secundario }\end{array}$ & Cámara trampa & - \\
\hline
\end{tabular}

C. centralis en Colombia se dio en 1898 (Bangs, 1900) y el más reciente en 2017 (Chacón-Pacheco et al., 2019).

Finalmente, Costa Rica tuvo el primer registro de $C$. centralis, en 1981. Hall (1981) informó que este armadillo se encuentra en todo el país, mientras que el reporte más reciente fue en 2009 (Saénz \& Carrillo, 2009), para un total de siete hallazgos. Esta escasez de avistamientos confirma que $C$. centralis es una especie rara, y por ende con poca información documentada, por lo que evidencia la necesidad de ser estudiada, para fundamentar planes de conservación. Con este nuevo reporte en el país, se hace constar que no solo se encuentra en ambientes naturales, sino también en hábitats fragmentados en donde se encuentran intercalados agroecosistemas como los de cultivo de café.

\section{AGRADECIMIENTOS}

Agradecemos a los productores Olman Ramírez y Gerardo Quirós por permitir realizar la investigación en sus agroecosistemas con café. Así como al Programa Jaguar-UNA y al Programa de Investigación de Recursos Naturales (PIRENA) de la Universidad de Costa Rica, Sede de Occidente por el préstamo de las cámaras trampas.

\section{REFERENCIAS}

Aguiar, J. (2004). The 2004 edentate species assessment workshop: species summary and species discussion. Edentata, 6, 3-26. DOI: 10.1896/1413-4411.6.1.3

Allen, J. A. (1904). Report on mammals from the district of Santa Marta, Colombia, collected by Mr. Herbert H. Smith: with field notes by Mr. Smith. Bulletin of the National Museum of Natural History, 20, 407-468.

Bangs, O. (1900). List of the mammals collected in the Santa Marta region of Colombia by W. W. Brown Jr. Proceedings of the New England Zoological Club, 1, 87-102.

Bard, S. (1855). Waikna; or, adventures on the Mosquito shore. New York, USA: Harper \& Brothers.

Cañón, S., \& Trujillo, F. (2014). Mastofauna. In: L. Báez y F. Trujillo (Eds.), Biodiversidad en Cerrejón. Bogotá, Colombia: Carbones de Cerrejón, Fundación Omacha, Fondo para la Acción Ambiental y la Niñez.

Carrillo, E., \& Wong, G. (1992). Registro y medidas de restos de un Cabassous centralis (Edentata: Dasypodidae) en el Parque Nacional Manuel Antonio, Quepos, Costa Rica. BRENESIA, 38, 153-154.

Carrillo, E., Wong, G., \& Sáenz, J. (2002). Mamíferos de Costa Rica. Santo Domingo, Heredia, Costa Rica: Instituto Nacional de Biodiversidad. 
Chacón-Pacheco, J., Ballesteros-Correa, J., \& Racero-Casarrubia, J. (2019). Primer registro en cámaras trampa del armadillo Coletrapo Cabassous centralis (Cingulata, Chlamyphoridae) en el Departamento de Córdoba, Colombia. Oecología Australis, 23(1), 150-155. DOI: 10.4257/oeco.2019.2301.14

CIGEFI (Centro de Investigaciones Geofísicas). (2019). Datos de la Estación Meteorológica de la Sede de Occidente, Universidad de Costa Rica. San Ramón, Alajuela: Universidad de Costa Rica.

Contreras, M., \& Yanguez, A. (2017). Caracterización de los usos de vertebrados silvestres en la comunidad de Quebrada Ancha, Colón (Panamá). CENTROS, 6(2), 18-30.

Corredor, G., Peralta, L., Castaño, J., Zuluaga, J., Henaos, B., Arango, M., ... Restrepo, A. (2004). The naked-tailed armadillo Cabassous centralis (Miller 1899): a new host to Paracoccidioides brasiliensis. Molecular identification of the isolate. Medical Micologya, 43(3), 275-280. DOI: $10.1080 / 13693780412331271090$

Cruz-Rodríguez, C., Larrota, L., González-Maya, J., Zarrate-Charry, D., Cepeda, A., Balaguera-Reina, S., ... Uribe-Castaño, C. (2011). New record for the northern naked-tailed armadillo Cabassous centralis (Cingulata: Dasypodidae) in tropical dry forest of the department of Sucre, Colombian Caribbean. Revista Mexicana de Mastozoología, 15, 3945. DOI: 10.22201/ie.20074484e.2011.1.1.11

Cuarón, A. D. (2005). Armadillo cola de zorro. En G. Ceballos \& G. Oliva (Eds.), Los mamíferos silvestres de México (pp. 116-17). México D.F.: CONABIO y Fondo de Cultura Económica.

Cuarón, A., March, I., \& Rockstroh, P. (1989). A second armadiIlo (Cabassous centralis) for the faunas of Guatemala and México. Journal of Mammalogy, 70, 870-871. DOI: $10.2307 / 1381731$

Delsuc, F., Gibb, G. C., Kuch, M., Billet, G., Hautier, L., Southon, J., ... Poinar, H. N. (2016). The phylogenetic affinities of the extinct glyptodonts. Current Biology, 26, 141-156. DOI: 10.1016/j.cub.2016.01.039

Díaz-N, J., \& Sánchez-Giraldo, C. (2008). Notable altitudinal range extension of the northern naked-tailed armadillo Cabassous centralis (Cingulata: Dasypodidae) in Colombia. BRENESIA, 69, 75-76.

Emmons, L., \& Feer, F. (1990). Neotropical rainforest mammals. A field guides. Chicago, Illinois, USA: University of Chicago Press.

Figueroa-De-León, A., Naranjo, E., \& Santos-Moreno, A. (2016). Registros de Cabassous centralis (Cingulata: Dasypodidae) en la Reserva de la Biosfera Montes Azules y sitios aledaño, Chiapas, México. Edentata, 17, 46-50. DOI: 10.2305/IUCN.CH.2016.EDENTATA-17-1.8

Gardner, A. L., Wetzel, R. M., Redford, K. H., \& Eisenberg, J. F. (2007). Order Cingulata. In A. L. Gardner (Ed.), Mammals of South America: Volume 1 Marsupials, Xenarthrans, Shrews, and Bats. Chicago, USA: University of Chicago Press.
Gennoways, H., \&Timm, R. (2003). The Xenarthrans of Nicaragua. Maztozoología Neotropical, 10(3), 231-253.

González-Zamora, A., Arroyo-Rodríguez, V., Gonzaléz-Di Pierro, A., Lombera, R., De La Peña-Cuellar, E., Peña-Mondragón, J., ... Stoner, K. (2011). The northern naked-tailed armadillo in the Lacandona rainforest, México: new records and potential threats. Revista Mexicana de Biodiversidad, 82, 581-586. DOI: 10.22201/ib.20078706e.2012.2.1248

Hall, R. (1981). The mammals of North America (Vol. II). New York, USA: John Wiley \& Sons.

Hayssen, V., Ortega, J., Morales-Leiva, \& Martínez-Mendez, N. (2012). Cabassous centralis (Cingulata: Dasypodidae). Mammalian Species, 45(898) ,12-17. DOI: 10.1644/898.1

Hoskins, H., Burdekin, O., Dicks, K., Salter, K., McCann, N., Jocque, M., ... Reid, N. (2018). Non-volant mammal inventory of Cusuco National Park, northwest Honduras: reporting the presence of Jaguar, Panthera once (Linnaeus, 1758) and demonstrating the effects of zonal protection on mammalian abundance. Check List, 5, 77-891. DOI: $10.15560 / 14.5 .877$

Ingles, G. (1953). Observation on Barro Colorado Island mammals. Journal of Mammalogy, 34, 266-268. DOI: 10.1093/ jmammal/34.2.266a

Juárez-López, R., Pérez López, M., Bravata-de la Cruz, Y., Jesús de la Cruz, A., Contreras-Moreno, F., Thornton, D., \& Hidalgo-Mihart, M. (2017). Range extensión of the northern naked-tailed armadillo (Cabassous centralis) in Southern México. Western North American Naturalist, 77(3), 398-403. DOI: 10.3398/064.077.0311

Mendoza-Durán, A. (2005). Armadillo. En G. Ceballos \& G. Oliva (Eds.), Los mamíferos silvestres de México (pp- 117-19). México D.F., México: CONABIO y Fondo de Cultura Económica.

Reid, F. (2009). A Field Guide to the Mammals of Central America and Southeast Mexico ( $2^{\text {nd }}$ Ed). New York, USA: Oxford University Press.

Rodríguez, J., \& Chinchilla, F. (1996). Lista de mamíferos de Costa Rica. Revista de Biología Tropical, 44(2), 877-890.

Rodríguez-Mahecha, J. V., Rueda-Almonacid, J. V., \& Hinojosa, T. D. G. (2008). Guía ilustrada de fauna del Santuario de Vida Silvestre Los Besotes, Valledupar, Cesar, Colombia. Bogotá, Colombia: Conservación Internacional.

Sáenz, C., \& Carrillo, E. (2009). Registro de Cabassous centralis (Cingulata; Dasypodidae) en la Reserva Forestal Pacuare y Reserva Indígena Nairi Awairi, Siquirres, Costa Rica. Brenesia, 71-72, 69-70.

Sánchez, R., \& Moya, M. (2018). Biodiversidad en fincas cafetaleras de Rincón de Mora, San Ramón, Alajuela, Costa Rica. Revista Pensamiento Actual, 31, 68-86. DOI: 10.15517/PA. V18I31.35666

Timm, R., Wilson, D., Clauson, B., LaVal, R., \& Vaughan, C. (1989). Mammals of the La Selva Braulio Carrillo complex, Costa Rica. Washington D.C., USA: U.S Department of the 
Interior Fish and Wildlife Service. DOI: 10.5962/bhl. title.86974

UICN. (2019). La Lista Roja de Especies Amenazadas de la UICN (Versión 2019-1). Recuperado de www.iucnredlist.org

Wetzel, R. (1980). Revision of the naked-tailed armadillos, genus Cabassous Mc Murtie. Annals of Carnegie Museum, 49, 323-357.
Wilson, D. (1983). Checklist of mammals. In D. H Janzen (Ed.), Costa Rican Natural History. Chicago, USA:The University of Chicago Press.

Woodman, N. (1998). Listado de mamíferos registrados durante mayo y junio de 1998 en San Ramón, Isla de Ometepe. Recuperado de http://vmaderas.tripod.com/inventarios/inventarios.html 